\title{
DIFERENCIAS EN RESULTADOS ACADÉMICOS ENTRE EDUCACIÓN TÉCNICO-PROFESIONAL Y HUMANISTA-CIENTÍFICA EN CHILE
}

\author{
Mauricio Farías, Rafael Carrasco ${ }^{1}$
}

\begin{abstract}
RESUMEN
Este estudio compara las diferencias en el rendimiento académico entre estudiantes de enseñanza media técnico-profesional y humanista-científica en Chile, usando propensity score matching y análisis de sensibilidad. Las estimaciones indican que los estudiantes de educación técnico-profesional obtienen en promedio 0,28 desviaciones estándar por debajo de estudiantes similares en educación humanista-científica. Adicionalmente, la evidencia muestra efectos diferenciados según nivel de logro inicial. Estos efectos alcanzan hasta 0,59 desviaciones estándar para alumnos con mayores habilidades académicas. Estos estudiantes podrían limitar seriamente sus oportunidades de acceso a educación superior al preferir educación técnico-profesional. El análisis de sensibilidad sugiere que la mayoría de estas diferencias son suficientemente grandes como para ser significativas incluso ante la posible presencia de variables importantes omitidas.
\end{abstract}

Palabras clave: educación técnica, educación media, propensity score matching

\section{DIFFERENCES IN LEARNING OUTCOMES BETWEEN TECHNICAL-PROFESSIONAL EDUCATION AND SCIENTIFIC- HUMANISTIC EDUCATION IN CHILE}

\begin{abstract}
This study analyzes differences in academic achievement among students enrolled in vocational education compared to students in academic education at the secondary level in Chile. Using OLS, propensity score matching and sensitivity analysis we examine test score differences between 10th and 12th grades in math and language. The study provides evidence that vocational education students perform, on average, 0.28 standard deviations below similar students in academic education. There is additional evidence of differentiated effects depending on the initial achievement levels. These effects range from 0.08 to 0.49 standard deviations for lower and higher achievers respectively. Finally, the sensitivity analysis suggests that most of these differences are important enough to be significant even in the presence of potentially strong omitted variables.
\end{abstract}

Keywords: vocational education, academic education, achievement, propensity score matching

1 Universidad de Stanford, California, EE.UU. Contacto: mauricio_farias@yahoo.com 


\section{DIFERENCIAS EN RESULTADOS ACADÉMICOS ENTRE EDUCACIÓN TÉCNICO-PROFESIONAL Y HUMANISTA-CIENTÍFICA EN CHILE}

\section{Introducción}

Durante la última década, la educación superior ha adquirido una gran relevancia internacional. La importancia de la ciencia y la tecnología en la economía ha crecido vertiginosamente, generando una mayor demanda de trabajadores calificados (Drori et al, 2003; Schoefer y Meyer, 2005). Al mismo tiempo, el aumento en las tasas de matrícula secundaria (OECD, 2008a) y la reducción en las tasas de retorno de este nivel educacional han trasladado a la educación terciaria la competencia por diferenciación y acceso a mejores oportunidades. Frente a estas dos dimensiones de productividad y diferenciación, aparecen como contrapartida los temas de eficiencia y equidad en la educación terciaria. El acceso a educación superior es uno de los tópicos que conecta directamente ambos temas: le eficiencia, por las posibles pérdidas de capital humano si el sistema de acceso tiene limitaciones importantes, y la equidad, por la posible existencia de barreras o facilitadores para grupos específicos de la población. En este contexto, el presente estudio examina la preparación de estudiantes matriculados en educación media técnico-profesional $\left(\right.$ ETP $\left.^{2}\right)$ en Chile, con el fin de evaluar el efecto de la provisión de ETP en las oportunidades de acceso a educación superior y posibles efectos en la equidad y eficiencia del sistema educativo.

Contrariamente a lo que mucha gente cree, la ETP es un importante camino hacia la educación superior en Chile. Por ejemplo, más del $70 \%$ de los padres de estudiantes de $2^{\circ}$ medio en el año 2006 esperaba que su hijo o hija alcanzara un título de educación terciaria. Alrededor del 35\% de estos estudiantes eligieron ETP. En

2 En adelante usaremos ETP para referirnos a la educación técnico-profesional a nivel de educación media. Nos referiremos a la educación científico-humanista en este mismo nivel educativo como EHC 
cifras del Ministerio de Educación (Mineduc 2010), casi el 46\% de los estudiantes de $3^{\circ}$ y $4^{\circ}$ medio eligió ETP. De los estudiantes de ETP, casi el 50\% continúan efectivamente en educación postsecundaria (Microdatos, 2009). Por lo tanto, para una proporción no despreciable de estudiantes de ETP la calidad de la preparación académica afecta directamente sus posibilidades futuras para la educación superior. Esto es particularmente cierto en un mundo que se mueve hacia un modelo de aprendizaje permanente, donde se espera volver a entrar al sistema educativo en reiteradas ocasiones en la vida.

Sin embargo, este no es solo un tema local sino global. En el mundo no existe un consenso claro sobre los efectos de la provisión de ETP y un intenso debate se desarrolla con relación a sus efectos en la educación y el mercado laboral (OCDE, 2008a; Hanushek y Wößmann, 2005). Incluso en los Estados Unidos, donde la provisión de ETP casi ha desaparecido (OCDE, 2008b), han surgido nuevas voces pidiendo el fortalecimiento de este tipo de educación (Symonds, 2011).

Al buscar evidencia internacional para entender el fenómeno se aprecia que la literatura se ha centrado en los efectos de la ETP en la empleabilidad o la retención de los estudiantes en el sistema educativo (OECD, 2008a; Meer, 2007). La literatura que analiza diferencias de rendimiento académico con la educación "humanista-científica" (EHC) es escasa y en la mayoría de los casos no se consideran los efectos producidos por las diferentes poblaciones que componen los grupos comparados (sesgo de selección). Este estudio espera contribuir con evidencia sobre las diferencias en los resultados académicos entre los estudiantes que asisten a programas de ETP y EHC en Chile.

La literatura señala que, si bien se podrían esperar efectos positivos de la ETP en la empleabilidad y la permanencia de los alumnos en el sistema educativo, el costo esperado de estudiar ETP es un efecto negativo en el rendimiento académico. La ETP podría reducir el nivel de instrucción y el logro de los estudiantes debido a una serie de razones, tales como programas de estudios menos exigentes, docentes con menor rendimiento, efecto par negativo, estigmatización y disminución de las expectativas (Arum y Shavit, 1995). 
Algunos autores exploran las diferencias en rendimiento entre EHC y ETP en la teoría o solo con pequeñas muestras (Oakes, 1985; Shavit y Muller, 2000). Otros abordan directamente la cuestión de una posible brecha de rendimiento entre los estudiantes de EHC y ETP, pero rara vez consideran el sesgo de selección (Lucas, 1999; Gamoran, 1987; OCDE, 2008b). Además, la mayor parte de esta literatura se refiere al sistema de tracking ${ }^{3}$ en los EE.UU. Mientras que la mayoría de los estudiantes de EE.UU. cuentan con un currículo flexible (libertad para elegir cursos orientados o no orientados a educación terciaria), en Chile y otros países, los estudiantes eligen o son clasificados en especialidades con programas más rígidos y diferenciados (diferentes planes de estudios, profesores y compañeros de clases). En este sentido, Lucas (1999) demostró que las asignaturas cursadas por los estudiantes en los EE.UU. son heterogéneas, dificultando su clasificación como estudiantes de ETP o EHC.

La escasez de esta literatura puede inducir a error en el debate sobre los efectos de ETP en comparación a los de EHC. Reducciones importantes en el rendimiento académico de los estudiantes ETP puede limitar sus posibilidades de continuar en la educación superior, no solo profesional sino también técnica. Además, podría dar lugar a pérdidas significativas en la acumulación de capital humano, tanto para cada individuo como también para toda la sociedad.

El objetivo de este estudio es cuantificar las diferencias en el rendimiento académico entre los estudiantes en EHC y ETP, para entender de mejor forma los efectos de la elección de ETP en las oportunidades de acceso a educación superior y posibles efectos en la equidad y eficiencia del sistema educativo. Para ello, sigue la cohorte de estudiantes de $8^{\circ}$ básico del año 2004 en Chile. Estos asisten a ETP o EHC en $3^{\circ}$ y $4^{\circ}$ medio y rinden la PSU al término del año 2008. En particular, se busca entender las consecuencias académicas para los estudiantes ETP que deciden someterse a las pruebas de admisión para la educación superior (PSU). El estudio responde a las siguientes preguntas:

3 Clasificación de los alumnos en cursos según nivel de desempeño. 
- ¿En qué medida alumnos comparables de EHC y ETP se diferencian en su desempeño en la PSU?

- ¿Varía esta diferencia según las características de los estudiantes (niveles de logro previo, de género, entre otros)?

- ¿Hay diferencias de brecha en los subsectores de matemática y lenguaje?

- ¿Cómo varía esta brecha para diferentes tipos de especialidades ETP (agrícola, comercial, eléctrica, industrial, marítimo y mecánica) en comparación con alumnos de EHC?

- ¿Hasta qué punto la elección de ETP limita las opciones de acceso a la universidad?

\section{Educación técnico-profesional y humanista- científica en Chile}

El sistema educacional chileno tiene características que lo hacen único e interesante de estudiar. El financiamiento de las escuelas en Chile emplea un sistema de quasi-vouchers, el cual paga una subvención directamente a las escuelas, de acuerdo con la matrícula y asistencia de los alumnos. Durante los años 2006 a 2008, el 93\% de la población estudiantil asistió a escuelas subvencionadas: el 45\% asistió a escuelas públicas y el $48 \%$ a escuelas privadas subvencionadas (Mineduc, 2010). El sistema favorece la competencia entre establecimientos y posibilita a los padres elegir el establecimiento educacional que consideren mejor para sus hijos.

La estructura del sistema durante los años de estudio era la siguiente $e^{4}$ : educación básica, grados 1-8 y educación media, grados 9-12. La reforma curricular de 1999 postergó la decisión de una especialidad hasta después del décimo grado. Esta se concentra principalmente en dos alternativas: educación humanístico-científica (EHC), que prepara a los estudiantes para la educación superior y la educación técnico-profesional (ETP), que teóricamente prepara

4 La estructura cambia con la aprobación de la Ley General de Educación en el año 2009, pasando a ed. básica: grados 1-6; ed. media: grados 7 a 12 . 
a los estudiantes para el mercado laboral ${ }^{5}$. La decisión no implica costos directos para los estudiantes y pueden cambiar su decisión después de haber optado por una especialidad. Como se mencionó con anterioridad aproximadamente $46 \%$ de los estudiantes de $3^{\circ}$ y $4^{\circ}$ medio eligió ETP. De estos, 38\% eligió la especialidad comercial, 36\% industrial, 18\% técnica, 7\% agrícola y 2\% marítima (Mineduc, 2010). Otro antecedente es que un poco más del $50 \%$ de los estudiantes asiste a establecimientos que solo proveen educación básica. Muchos alumnos eligen su especialidad al terminar $8^{\circ}$ básico debido a la existencia de establecimientos de educación media que imparten solo una especialidad. Formalmente, el currículum de $1^{\circ}$ y $2^{\circ}$ medio es el mismo para ambas especialidades y los alumnos solo deciden al terminar $2^{\circ}$ medio. Este estudio considera que la decisión se toma al terminar $2^{\circ}$ medio, ya que si la decisión fue tomada antes, debe ser confirmada de todas formas al terminar $2^{\circ}$ medio.

La ley permite a las escuelas definir su propio plan de estudios condicional al cumplimiento de ciertos requisitos. En el caso del currículo, las escuelas de ETP deben asignar al menos 600 horas de instrucción por año a cursos de formación técnico-profesional. En casos como la especialidad comercial de ETP, estas horas pueden destinarse a temas relacionados con matemática o lenguaje, lo que podría afectar positivamente el rendimiento de los alumnos en la PSU. Aunque es esperable un mayor rendimiento académico en los test de los alumnos de EHC sobre ETP, este estudio busca cuantificar la magnitud de esta diferencia así como algunas de sus características.

Después de la educación media, todos los estudiantes tienen la opción de acceder a la educación superior, ya sea universitaria o técnica. La admisión a las carreras de las universidades más importantes es competitivo sobre la base del puntaje en la PSU. En general, las carreras técnicas no requieren puntajes en la PSU. En 2008, alrededor del 65\% de los estudiantes que se graduaron de la enseñanza secundaria rindieron la PSU y aproximadamente el 30\%

5 La ETP está aún definida en la ley como una educación de carácter terminal. Sin embargo, muchos establecimientos educacionales de ETP preparan a sus estudiantes para educación superior. 
de ellos provenían de la ETP. Este grupo corresponde a alrededor del $46 \%$ de los estudiantes de $4^{\circ}$ medio matriculados en un programa de ETP en el año 2008. En contraste, alrededor del 88\% de los estudiantes matriculados en un programa de EHC rindieron la PSU. Aproximadamente el 47\% de los estudiantes en ETP continuaron sus estudios en educación superior en el año 2003 (Microdatos, 2009). De este grupo, al menos el 50\% intentó obtener un título universitario. No es claro cuántos no lo intentaron por bajo rendimiento.

\section{Revisión de la literatura}

La literatura sugiere diferentes razones para explicar la variación en los niveles de logro entre los estudiantes de ETP y EHC. Primero, podrían existir disparidades en las condiciones de aprendizaje. Las escuelas de ETP, por ejemplo, ofrecerían un programa más restringido (Hallinan y Kubitschek, 1999). Al dedicar más tiempo a la formación específica para el trabajo, reducen el tiempo dedicado a las habilidades académicas. Uno de los aspectos en que no existe mayor claridad en este sentido es cómo los cursos ETP podrían afectar los aprendizajes en temas básicos como lenguaje, matemática o ciencia. Adicionalmente, las bajas expectativas de los docentes en cuanto al rendimiento intelectual de los alumnos podrían reducir el alcance del plan definido para los estudiantes de ETP (Metz, 1978; Oakes, 1985). Por otra parte, como los programas de ETP no están orientados a la educación superior, es probable que solo enseñen parcialmente el material necesario para las pruebas de admisión de la educación superior (Arum y Shavit, 1995). Por último, los mejores profesores enseñarían en los cursos más exigentes (Finley, 1984; Oakes, 1985).

Una segunda razón se refiere a diferencias en los tipos de estudiantes que asisten a ETP o EHC. Por ejemplo, los estudiantes que asisten a EHC podrían estar más motivados, tener mayores expectativas, o provenir de familias que brindan más apoyo o son más preocupadas de los logros educativos. Los estudiantes más motivados y capacitados pueden mejorar el rendimiento de sus compañeros mediante efectos positivos entre pares, mientras que los estudiantes menos motivados y calificados podrían reducir el 
rendimiento de sus compañeros (Coleman et al, 1966; Hoxby, 2000; Sacerdote y Marmaros, 2005). Otra de las características importantes es el nivel socioeconómico de los estudiantes (SES). El SES estaría relacionado con mayor capacidad de apoyo, mayor nivel educativo, y mayor preocupación por los resultados, por lo que un mayor SES está asociado a un mayor rendimiento. Un tercer elemento relacionado a las características de los estudiantes son sus expectativas. Es esperable que la ETP reúna a los estudiantes con menores expectativas. Esta situación podría llevar a reducir aún más las propias expectativas así como las de los demás (profesores, apoderados, etc.). Vanfossen et al. (1987) encontraron evidencia de efectos positivos de la especialidad académica (ECH) en las expectativas. También, encontraron efectos positivos de las expectativas en el rendimiento estudiantil.

La literatura que aborda la estimación de la brecha en resultados académicos entre la ETP y EHC es escasa. La OCDE (2005), en un análisis comparativo de países que utilizan PISA 2000, encontró que los estudiantes de ETP en la mayoría de los países obtenían puntuaciones significativamente inferiores a las de estudiantes de EHC. Sin embargo, la metodología utilizada no aborda el sesgo de selección. La generalidad de los otros estudios que estiman esta brecha provienen de EE.UU. En esta literatura, las comparaciones de los resultados de las pruebas mostrarían diferencias estadísticamente significativas entre aquellos que toman cursos académicos y los que toman cursos técnicos.

Utilizando una muestra longitudinal, Gamoran (1987) encontró una diferencia en rendimiento entre los EHC y ETP de 0,4 desviaciones estándar (DE) en matemática y 0,14 DE en escritura. Para ello utilizó MCO e incluyó logros pasados, características individuales y de escuela. Sin embargo, no consideró el efecto de variables no observadas ni cambios en la muestra. Gamoran y Mare (1989) utilizaron un modelo que en su conjunto estima la probabilidad de ser colocados en una especialidad en particular, el efecto de esta en el rendimiento estudiantil, y el mismo efecto si los estudiantes hubieran optado por la especialidad alternativa. Ellos encontraron que la especialidad causa una diferencia en el rendimiento en matemática 
de 0,2 DE. Además, encontraron que el sesgo de selección era casi insignificante cuando se incluían los controles suficientes, incluyendo resultados de mediciones anteriores. No es claro cómo se abordan las posibles variables omitidas. Comparando diferentes modelos, Lucas (1999) encontró que los que incluyen la especialidad elegida por el estudiante alcanzaban un mayor poder explicativo que los que no, concluyendo que la especialidad explica los resultados en las mediciones. Carbonaro y Gamoran (2002), utilizando un modelo de dos niveles y tres puntos en el tiempo, encontraron una diferencia de alrededor de 0,1 DE para escritura. Por último, Carbonaro (2005), utilizando un modelo de MCO, encontró diferencias de 0,33 DE en matemática.

En suma, los estudios anteriores no analizan convincentemente el sesgo de selección. Este estudio contribuye a la literatura ya existente enfrentando el potencial de sesgo de selección mediante el uso de propensity score matching y análisis de sensibilidad. Por tanto, el estudio se aproxima de mejor forma a identificar el efecto causal que la ETP tiene en el rendimiento académico de los estudiantes en Chile.

\section{Marco teórico}

La figura 1 presenta un diagrama que contiene los factores más importantes que afectan la elección de la especialidad y los logros académicos de los estudiantes, agrupados en tres niveles: estudiante, establecimiento y entorno del establecimiento (Gamoran, 1987). El nivel del estudiante incluye características del alumno (género, raza, etc.) y de sus familias (escolaridad de sus padres, ingresos del hogar, etc.). También, incluye resultados académicos en mediciones anteriores, el concepto de capital cultural en la familia (Bourdieu y Passeron, 1979), y las expectativas de los estudiantes (Kelly y Price, 2009). Se utilizará la variable de libros en el hogar como un proxy para capital cultural (Carnoy, 2007). 
Figura 1. Marco conceptual

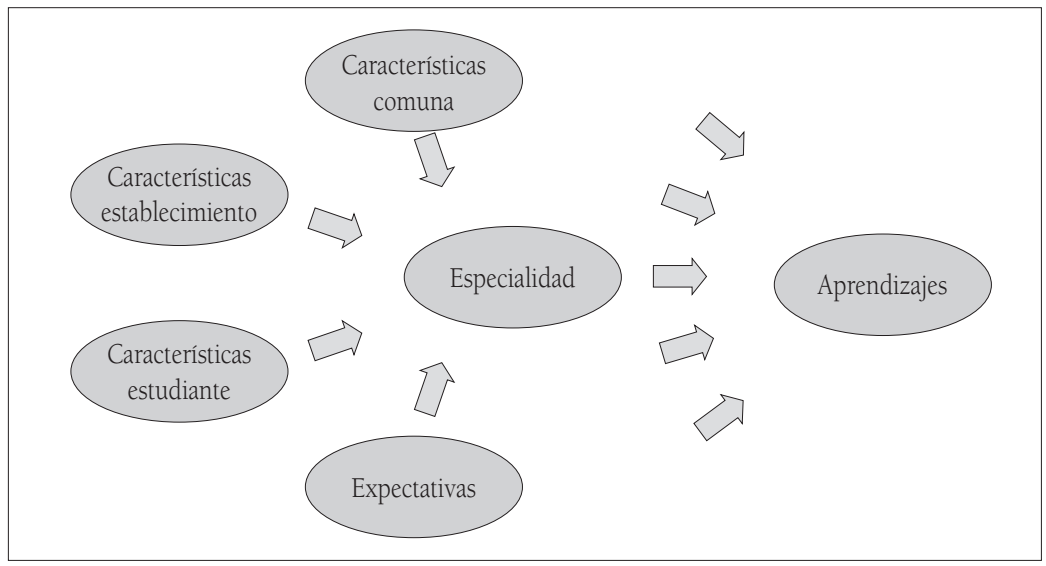

Basado en Gamoran 1987.

Los factores respecto del establecimiento educacional incluyen características de la escuela que afectan las decisiones de los estudiantes, ya sea debido a las restricciones formales o para el entorno en el que se lleva a cabo el proceso educativo (tipo de administración de la escuela, nivel socioeconómico promedio del alumnado, tamaño de la escuela, etc.). Con relación al entorno se consideran variables como el nivel socioeconómico de la comuna, la ruralidad y el número de escuelas existentes, entre otros.

Controlando por los factores descritos, el estudio busca establecer el efecto en resultados académicos de recibir ETP. El tratamiento "ETP" considera los dos años que un estudiante pasa en una escuela de ETP. Este tratamiento se basa en un programa que conduce a un certificado de formación profesional. Incluye no solo el plan de estudios de la especialidad, sino también otras características que difieren con la EHC como los maestros, los periodos de formación y las diferentes instalaciones. El grupo de control abarca a los estudiantes que eligen EHC.

\section{Datos}

En este estudio se aprovechan datos que por primera vez es posible reunir en Chile. La información incluye resultados de las pruebas 
del Sistema de Medición de la Calidad, SIMCE de $8^{\circ}$ básico (2004) y segundo medio (2006), además de la Prueba de Selección Universitaria, PSU 2008. El SIMCE aplica mediciones basadas en el currículo en casi la totalidad de los alumnos correspondientes a los niveles medidos cada año. Esta prueba estima por lo menos habilidades de lenguaje y matemática (ver Meckes y Carrasco, 2010). En 2004, junto a la medición también se encuestó a los padres, estudiantes y maestros de matemática y lenguaje. En 2006, solo se incluyó encuestas a los padres. Es importante mencionar que algunas variables (como el ingreso familiar, la escolaridad promedio de los padres y el número de libros en casa) que se encontraban incompletas para el año 2006, fueron imputadas utilizando datos de 2004.

La PSU es un examen anual que se toma al final del año escolar y es un requisito para el ingreso a la mayoría de las universidades del país. La prueba incluye tanto los resultados de los distintos subsectores (matemática, lenguaje, ciencia e historia y ciencias sociales), como información de características de los estudiantes. Si bien esta busca seleccionar estudiantes, considera en su diseño los contenidos curriculares definidos para la educación general (Comité Técnico Asesor, 2010), lo que permite la comparación entre PSU y SIMCE. La mayor parte del análisis se realiza utilizando el promedio de los puntajes estandarizados de matemática y lenguaje.

\section{Métodos}

En esta sección se presentan los principales obstáculos para responder a las preguntas de investigación y las metodologías utilizadas para hacer frente a estos problemas potenciales.

\section{Posibles sesgos}

Como se mencionó anteriormente, uno de los principales obstáculos para la inferencia causal de las diferencias entre alumnos de ETP y EHC en su rendimiento en la PSU es el sesgo de selección. Existen por lo menos dos fuentes para esto: los alumnos se autoseleccionan en los distintos programas (EHC y ETP) y también se autoseleccionan para la rendición de la PSU. 
En el primer caso, los estudiantes pueden autoseleccionarse en la especialidad (ETP o EHC) en función de su capacidad autopercibida, de sus expectativas y sus motivaciones. Por lo tanto, los dos grupos no son necesariamente comparables. Los estudiantes que asisten a ETP podrían ser diferentes en muchas formas, tanto observables como no observables, en comparación con los estudiantes que asisten a la EHC. La elección de especialidad por parte de los estudiantes podría verse afectada por razones que también estén relacionadas con el rendimiento. En particular, la motivación y las habilidades individuales son variables que comúnmente se reconocen como no observadas y que afectan tanto la elección de especialidad como el rendimiento. Es esperable que los estudiantes más motivados y con mayores habilidades elijan EHC para obtener la mejor preparación disponible en su camino a la educación superior. Por lo tanto, los estudiantes de EHC podrían tener un mayor desempeño no solo por diferencias en el programa sino también por la composición del alumnado.

En este estudio, los puntajes del SIMCE y las expectativas que los padres tienen sobre los estudiantes pueden servir como un buen proxy de la capacidad y la motivación, respectivamente. Estas dos variables en conjunto con otras variables relacionadas a las características de los estudiantes, sus familias, los establecimientos y el entorno, proporcionan un buen conjunto de controles de las diferencias entre las poblaciones de ETP y EHC. En efecto, los potenciales factores de confusión que podrían afectar la selección de especialidad y el logro de los estudiantes en la PSU probablemente son en su mayor parte controlados por este conjunto de variables. Sin embargo, una propensión especial hacia la educación académica o hacia el trabajo manual en el caso de ETP, que puede estar relacionado con el capital cultural, la autodisciplina, o una motivación extra para los dos últimos años de la escuela, podrían estar presentes y sesgar los resultados.

La segunda fuente de sesgo también es importante porque puede afectar la estimación de la brecha de puntajes entre alumnos de ETP y EHC al momento de proyectar los resultados desde la muestra a la población completa. Incluso, la autoselección de alumnos de EHC y ETP pueden llevar a sesgos en la estimación para la población 
observada. Por ejemplo, es esperable que solo los estudiantes más preparados, motivados y calificados de ETP rindan la PSU; mientras es esperable que la mayoría de los alumnos de EHC tomen el examen. Esta situación podría producir una subestimación de la brecha de rendimiento entre los dos grupos.

Esta segunda fuente de sesgo también incluye la deserción no aleatoria de la muestra cuando se estudia el efecto del tratamiento en toda la población. Un análisis rápido podría sugerir una mayor deserción de estudiantes de ETP, ya que esta población podría tener menor capacidad. En una muestra aleatoria o balanceada, en cambio, podría darse el caso que más estudiantes de EHC abandonen, ya que se enfrentan a un currículo más exigente. De todas formas, el sesgo de selección en la PSU es mayor y absorbe el que se produce por deserción.

Por último, otro posible sesgo puede afectar el estudio: no se conocen las motivaciones de los alumnos al momento de rendir la $\mathrm{PSU}^{6}$. Debido a que en muchas carreras técnicas de educación superior o incluso en algunas carreras universitarias se exige solo PSU rendida, las diferencias en motivación podrían afectar las estimaciones. Es posible argumentar que más estudiantes de ETP podrían estar optando a estas alternativas académicas que solo tienen requisito. El supuesto usado aquí es que la propensión a una carrera que solo requiere PSU rendida depende en gran medida de factores tales como el ingreso familiar per cápita, la escolaridad de los padres, las expectativas y el desempeño anterior. Si se considera que se realiza el matching con estas mismas variables, se espera que la propensión a asistir a una carrera que solo requiera PSU esté balanceada entre ambos grupos después del matching y, por tanto, no afecte los resultados.

Para estimar el efecto causal de ETP en el aprendizaje de los alumnos el estudio enfrenta el sesgo de selección con las siguientes metodologías:

6 La asistencia a preuniversitario también podría confundir los resultados. Se tuvo acceso a una muestra de 1.000 estudiantes de una encuesta representativa nacional que incluía la variable de asistencia a un preuniversitario. Se realizaron análisis similares a los aquí expuestos. Los resultados variaron en un modesto 0,01 SD para el promedio de la PSU. 


\section{Análisis MCO}

Se realizan estimaciones MCO para tener un análisis primario de los datos. Se excluyen de la población de análisis a los estudiantes que asisten a las escuelas privadas no subvencionadas, ya que solo una porción muy pequeña de los estudiantes asisten a ETP (menos del 1\%). El modelo a analizar es el siguiente:

$Y_{t}=\beta_{0}+\beta_{E T P} E T P+\beta_{E T P X Y} E T P X Y_{t-1}+\beta_{Y t-1} Y_{t-1}+\beta_{Y t-2} Y_{t-2}+\gamma X+\varepsilon$

donde "Yt" es el promedio estandarizado de la PSU de lenguaje y matemática 2008, "Yt-1" es el SIMCE de 2006, y "Yt-2" el SIMCE 2004. "ETP" es una variable que indica asistencia a un programa escolar ETP en tercero y cuarto medio; " $X$ " es un vector de covariables que incluye características de estudiantes, familias, escuelas y comunas. Se incluye un término de interacción (ETPXY) entre el ETP y el SIMCE 2006, ya que se espera que el efecto del tratamiento pueda variar con el nivel académico. Los errores se agrupan en la escuela para hacer frente a posibles correlaciones de las variables dentro de ellas.

\section{Propensity score matching}

La principal estrategia utilizada es el propensity score matching. Basándose en variables observables, se puede estimar la probabilidad de un alumno de asistir a ETP (propensity score). Con esta propensión se repondera el grupo control de tal forma de hacerlo comparable al grupo tratamiento. Rosenbaum y Rubin (1983) demostraron que la comparación del grupo tratamiento y control es no sesgada si el tratamiento es ignorable en un determinado valor del propensity score. Además, matching solo funciona en la zona donde los dos grupos son comparables $^{\top}$ (región de soporte común). Estas dos características son especialmente útiles en nuestro estudio. Primero, se sabe que más estudiantes de EHC rinden la PSU y se espera que los estudiantes más preparados de ETP hagan lo mismo, por lo tanto, el proceso de matching equilibra las características observadas de los grupos creando dos grupos comparables. Segundo, debido a que algunos estudiantes

7 Esto también implica que el efecto que estimamos es un resultado promedio solo en la población tratada y sus casos comparables. 
que optan por EHC podrían ser diferentes de los que optan por ETP en términos de capacidades, expectativas y nivel socioeconómico, restringir el análisis a la zona de soporte común permite eliminar los estudiantes que no tienen un contrafactual.

Matching asume independencia condicional, lo que significa que ni el tratamiento ni los resultados están correlacionados con las mismas variables no observables.

La crítica más común que se debe enfrentar aquí es que los grupos en realidad no son comparables y que el supuesto de independencia condicional es demasiado marcado para ser sostenido (Duflo et al., 2007). Dehejia y Wahba (2002) utilizando un diseño experimental como comparación, demostraron que matching reduce el sesgo con éxito cuando se corrige por variables observables. Otros autores también justifican esta estrategia, en particular, cuando la calidad del matching es evaluada y cuando se utiliza análisis de sensibilidad para ver el posible efecto de una variable omitida (Caliendo y Kopeinig de 2008, Reardon, Cheadle y Robinson, 2009; Rosenbaum, 1986; Rosenbaum y Rubin, 1983). Asumiendo que al controlar por el propensity score también se controla por variables no observables (Rosenbaum y Rubin, 1983), esta metodología elimina el sesgo de selección al inicio del tratamiento. El análisis de sensibilidad (Rosenbaum, 1986) ayudará a comprender la variabilidad de los resultados si existe alguna variable importante que ha sido omitida. Se utiliza el algoritmo conocido como kernel biweight con un calibre de 0,0001 y un máximo de diez casos dentro del calibre. El modelo utilizado para calcular el puntaje de propensión fue el siguiente:

$$
\mathrm{TP}=\beta_{0}+\beta_{\mathrm{CPi}} \mathrm{CP}+\AA_{\mathrm{Yt}-1} \mathrm{Yt}-1+\beta_{\mathrm{EX}} \mathrm{EX}+\varepsilon(2)
$$

donde TP es una variable dummy para los que tomaron ETP; CP es un vector que contiene las características personales (el mejor modelo considera el sexo, el promedio de escolaridad de los padres y su cuadrado, sus ingresos per cápita estandarizados, su cuadrado y su cubo, y el número de libros en casa); Yt-1 es un vector que contiene las puntuaciones anteriores del SIMCE $(2004,2006$ y el cuadrado de 
2006); por último, EX contiene las expectativas de los padres sobre el futuro de los estudiantes.

Se desarrolla el matching para dos muestras. La primera de ellas ("muestra 1") incluye solo a los estudiantes que rindieron el examen SIMCE 2004 en el octavo grado, el SIMCE 2006 en el décimo grado, y la PSU el 2008 al finalizar el duodécimo grado. En esta muestra también se excluyen los estudiantes de establecimientos privados no subvencionados. Esta es la muestra para la cual se reportan resultados. Con el fin de incluir más casos y estudiar el sesgo por selección de muestra, también se realizó el matching para todos los estudiantes que rindieron ambas pruebas SIMCE 2004 y 2006, incluyendo aquellos que no rindieron la PSU en 2008 ("muestra 2").

Es importante hacer una observación acerca de la validez externa. En este caso, el tratamiento no está definido para todos los estudiantes que se inscribieron en ETP, sino para aquellos estudiantes que se inscribieron en ETP y rindieron la PSU. Esto limita la población de inferencia causal a aquellos que rindieron esta prueba.

Para comprobar el balance entre el grupo control y el de tratamiento después del proceso de matching, se utilizan análisis gráficos y modelos MCO. En el primer caso, se incluye el gráfico del propensity score pre y post matching tanto para el grupo control como para el grupo bajo tratamiento. En el segundo caso, se incluyen las regresiones para cada variable contra el tratamiento, pre y post matching.

\section{Análisis de sensibilidad}

Con el fin de evaluar la solidez de los resultados, se realizó un análisis de sensibilidad (Reardon, Cheadle y Robinson, 2009; Rosenbaum, 1986). Este supone que una variable importante, un factor de confusión, no se observa y que su omisión produce un sesgo. Entonces, se estima la magnitud de ese posible sesgo. En un análisis conservador, se asume que el sesgo de esta variable es equivalente al mayor efecto posible estimable de las variables observadas. En particular, se estima su efecto como el producto 
del mayor coeficiente obtenido por las covariables estandarizadas, y la mayor diferencia entre los grupos de control y tratamiento de cada covariable. El sesgo estimado es descontado del efecto del tratamiento para probar la robustez de las conclusiones. Sin embargo, en este caso es evidente que las variables más importantes para predecir el puntaje PSU son los resultados en las pruebas anteriores $(2004,2006)$. Parece razonable considerar el más alto coeficiente de una variable sin tener en cuenta las puntuaciones anteriores de la prueba. Se presentan los resultados para las estimaciones pre y post matching.

\section{Extensión del análisis: efectos diferenciados}

Aprovechando el trabajo realizado de matching, se extiende el análisis sobre tres puntos que parecen interesantes. En primer lugar, se utilizan regresiones por cuantiles para el modelo (4) con el fin de explorar heterogeneidad en los posibles efectos del tratamiento en diferentes puntos de la distribución de resultados de la PSU. En segundo lugar, se estiman los efectos de las diferentes especialidades en la ETP. Con esta intención, se reemplaza el tratamiento ( $\mathrm{T}$ ) por variables dummy para distintas especialidades: comercial, industrial, agrícola, mecánica, y marítima. Se utiliza la EHC como base de comparación. Finalmente, se realizan regresiones por separado para matemática y lenguaje en la PSU.

\section{Cotas y selección de muestra}

Para hacer frente a la selección de muestra existente en la PSU, se analiza por deciles del SIMCE 2006, ya que el proceso de autoselección presenta una alta correlación con esta variable. Este paso permite obtener una imagen más nítida del grado de selección y la validez de las conclusiones de cada decil (ver tabla 1). Un mayor porcentaje de estudiantes de ETP no rindieron la PSU en cada decil. La figura 6 (ver anexo) muestra que los grupos de tratamiento y control tienen una distribución similar en cuanto a quienes rindieron o no la PSU, con una proporción ligeramente mayor de alumnos con menor puntaje para los estudiantes de ETP que no rindieron la PSU. 
104 DIFERENCIAS EN RESULTADOS ACADÉMICOS ENTRE EDUCACIÓN TÉCNICOPROFESIONAL Y HUMANISTA-CIENTÍFICA EN CHILE - M. Farías, R. Carrasco

Tabla n. $^{\circ}$ 1. Estudiantes que no rindieron la PSU 2008 por decil del SIMCE 2006 y tipo de programa educativo

\begin{tabular}{|c|c|c|c|c|c|c|c|c|c|c|c|c|}
\hline \multicolumn{13}{|c|}{ Decil Simce 2006} \\
\hline & \multirow[t]{2}{*}{ Rindió PSU } & & & & & & & & & & & \\
\hline & & 1 & 2 & 3 & 4 & 5 & 6 & 7 & 8 & 9 & 10 & Total \\
\hline \multirow{4}{*}{ EHC } & No & 4,393 & 3,880 & 3,434 & 3,265 & 2,794 & 2,512 & 2,219 & 1,806 & 1,376 & 843 & 26,522 \\
\hline & Sí & 3,121 & 4,025 & 4,843 & 5,605 & 6,773 & 7,803 & 9,363 & 11,117 & 13,676 & 17,526 & 83,852 \\
\hline & Subtotal & 7,514 & 7,905 & 8,277 & 8,870 & 9,567 & 10,315 & 11,582 & 12,923 & 15,052 & 18,369 & 110,374 \\
\hline & $\%$ No PSU & $58 \%$ & $49 \%$ & $41 \%$ & $37 \%$ & $29 \%$ & $24 \%$ & $19 \%$ & $14 \%$ & $9 \%$ & $5 \%$ & $24 \%$ \\
\hline \multirow{4}{*}{ ETP } & No & 11,953 & 10,898 & 9,906 & 8,907 & 7,707 & 6,620 & 5,278 & 4,111 & 2,611 & 963 & 68,954 \\
\hline & Sí & 2,978 & 3,641 & 4,261 & 4,667 & 5,170 & 5,509 & 5,584 & 5,410 & 4,781 & 3,112 & 45,113 \\
\hline & Subtotal & 14,931 & 14,539 & 14,167 & 13,574 & 12,877 & 12,129 & 10,862 & 9,521 & 7,392 & 4,075 & 114,067 \\
\hline & $\%$ No PSU & $80 \%$ & $75 \%$ & $70 \%$ & $66 \%$ & $60 \%$ & $55 \%$ & $49 \%$ & $43 \%$ & $35 \%$ & $24 \%$ & $60 \%$ \\
\hline & Total & 22,445 & 22,444 & 22,444 & 22,444 & 22,444 & 22,444 & 22,444 & 22,444 & 22,444 & 22,444 & 224,441 \\
\hline \multicolumn{2}{|c|}{ \% No rindió PSU } & $73 \%$ & $66 \%$ & $59 \%$ & $54 \%$ & $47 \%$ & $41 \%$ & $33 \%$ & $26 \%$ & $18 \%$ & $8 \%$ & $43 \%$ \\
\hline
\end{tabular}

Note: Decil 1 considera los estudiantes con los rendimientos más bajos.

A continuación se acotan los resultados mediante la reducción de la muestra según criterios que puedan dar un sentido claro a las cotas que se obtiene (Angrist et al., 2004). Para un límite superior de la brecha de puntaje, se estima en cada decil el porcentaje de estudiantes que no rindieron la PSU en ETP. Se reduce la proporción de estudiantes de EHC hasta el mismo porcentaje de estudiantes de ETP, sacando a los estudiantes de EHC con las puntuaciones más bajas en el SIMCE 2006. De esta manera se consideran solo los mejores alumnos de EHC con lo que se genera un límite superior para la estimación. Para la cota inferior se realiza un procedimiento similar pero eliminando a los estudiantes de EHC con mayor rendimiento.

\section{Balance de la muestra}

La figura 2 en el anexo presenta las regiones de soporte común a los dos grupos. Es evidente que la coincidencia entre las dos regiones es casi completa. La zona de sobreposición representa el 97,2\% del total de la muestra.

La figura 3 en el anexo presenta la densidad del propensity score antes y después del proceso de matching. Cuando se evalúa el balance de la muestra después del matching, se puede ver en la figura 3 que 
el propensity score adquiere una distribución casi idéntica entre los grupos tratamiento y control. Además, la mayoría de las diferencias en las covariables incluidas en el matching desaparecen (tabla 2). Algunas covariables aun mantienen diferencias significativas tras el matching. Esto ocurre en particular para las variables dummy, como "mujer" o la "expectativa de título de formación profesional en educación superior". Estas diferencias son mucho menores que las existentes antes del matching. La variable continua que sigue siendo significativa es la escolaridad de los padres, pero se reduce su coeficiente en dos órdenes de magnitud. En el anexo (figura 4) se presentan los gráficos de la distribución de grupos de tratamiento y de control, pre y post matching, para la variable "escolaridad de los padres".

Tabla n. ${ }^{\circ}$ 2. Evaluando el matching: diferencia promedio entre el grupo tratamiento y control para cada covariable pre y post matching.

\begin{tabular}{|c|c|c|c|c|c|c|c|c|c|}
\hline & SIMCE06 & SIMCE04 & Libros & Mujer & Escolaridad & Exp. ETP & Exp. ET-ES & Ypc06 & Prop. Score \\
\hline $\begin{array}{l}\text { Pre-matching } \\
\mathrm{T}\end{array}$ & \begin{tabular}{|c|}
$-0,379 * *$ \\
$(0,005)$
\end{tabular} & $\begin{array}{c}-0,356^{* *} \\
(0,005)\end{array}$ & $\begin{array}{c}-10,257^{* *} \\
(0,207)\end{array}$ & $\begin{array}{c}-0,040^{* * *} \\
(0,003)\end{array}$ & 9) & $\begin{array}{c}-0,047 * * * \\
(0,001)\end{array}$ & $\begin{array}{l}0,096^{* *} \\
(0,002)\end{array}$ & $\mid \begin{array}{r}-0,424 * * \\
(0,005)\end{array}$ & $\begin{array}{l}0,184^{* *} \\
(0,001)\end{array}$ \\
\hline \begin{tabular}{|l|l}
$\mathrm{N}$ \\
$\mathrm{R}-2$ \\
\end{tabular} & $\begin{array}{c}105,218 \\
0,048\end{array}$ & \begin{tabular}{|c|}
105,218 \\
0,044 \\
\end{tabular} & $\begin{array}{c}105,218 \\
0,023 \\
\end{array}$ & \begin{tabular}{c|}
105,218 \\
0,002 \\
\end{tabular} & $\begin{array}{c}105,218 \\
0,087\end{array}$ & $\begin{array}{c}105,218 \\
0,012\end{array}$ & $\begin{array}{c}105,218 \\
0,017 \\
\end{array}$ & $\begin{array}{c}105,218 \\
0,058 \\
\end{array}$ & $\begin{array}{c}105,218 \\
0,180 \\
\end{array}$ \\
\hline $\begin{array}{l}\text { Post-matching } \\
\mathrm{T}\end{array}$ & $\begin{array}{c}0,000 \\
(0,005) \\
\end{array}$ & $\begin{array}{c}-0,007 \\
(0,005) \\
\end{array}$ & $\begin{array}{l}-0,252 \\
(0,170) \\
\end{array}$ & $\begin{array}{c}-0,011^{* * *} \\
(0,003) \\
\end{array}$ & $\begin{array}{c}-0,051^{* *} \\
(0,018) \\
\end{array}$ & $\begin{array}{c}0,001 \\
(0,001)\end{array}$ & $\begin{array}{c}-0,010^{* *} \\
(0,003) \\
\end{array}$ & $\begin{array}{c}0,001 \\
(0,003) \\
\end{array}$ & $\begin{array}{c}0,000 \\
(0,001)\end{array}$ \\
\hline $\begin{array}{l}\mathrm{N} \\
\mathrm{R}-2\end{array}$ & $\begin{array}{c}105,218 \\
0,000 \\
\end{array}$ & \begin{tabular}{|c|}
105,218 \\
0,000 \\
\end{tabular} & $\begin{array}{c}105,218 \\
0,000 \\
\end{array}$ & \begin{tabular}{|c|}
105,218 \\
0,000 \\
\end{tabular} & $\begin{array}{c}105,218 \\
0,000 \\
\end{array}$ & $\begin{array}{c}105,218 \\
0,000 \\
\end{array}$ & $\begin{array}{c}105,218 \\
0,000 \\
\end{array}$ & $\begin{array}{c}105,218 \\
0,000 \\
\end{array}$ & $\begin{array}{c}105,218 \\
0,000 \\
\end{array}$ \\
\hline
\end{tabular}

Nota: Diferencias significativas entre grupos: $+\mathrm{p}<0,10,{ }^{*} \mathrm{p}<0,05,{ }^{* *} \mathrm{p}<0,01$. Errores estándar entre paréntesis.

Después del proceso de matching, parece seguro avanzar hacia el análisis de la diferencia entre los grupos tratamiento y control. El gran número de observaciones con que se cuenta y el hecho de que la mayoría de las diferencias aparecen en las variables categóricas, permite justificar las pequeñas diferencias encontradas. Con el fin de controlar por las diferencias que siguen siendo estadísticamente significativas, se incluyen todas las variables consideradas en el propensity score en los modelos utilizados para estimar los efectos del tratamiento. Por último, el equilibrio obtenido en las variables más importantes (es decir, resultados de exámenes anteriores) y el análisis visual de los gráficos generan confianza en el matching realizado. 


\section{Resultados}

\section{Efecto de la ETP}

Al estimar la brecha de puntajes en la PSU entre estudiantes de ETP y EHC en 2008 (incluidos los controles), se obtiene una diferencia de -0,28 DE (tabla 3). Este resultado se mantiene relativamente estable para las distintas metodologías y muestras. El coeficiente para el término de interacción entre el tratamiento y el puntaje SIMCE 2006 es -0,075. Como el SIMCE 2006 se mueve para alumnos de ETP en un rango entre -3,15 y 2,79, y teniendo en cuenta los términos del tratamiento y la interacción, el efecto total del tratamiento se mueve entre 0,08 y 0,49 DE. El puntaje promedio de la PSU es de 512 puntos y una DE equivale a 106,6 puntos. En consecuencia, los estudiantes ETP obtienen un puntaje en la PSU entre 8 y 52 puntos menos que estudiantes similares de EHC. En promedio, el puntaje es menor en 30 puntos. En términos de percentil, una diferencia de 0,50 DE equivaldría a que un estudiante en el percentil 50 se traslade al percentil 68 y un estudiante del percentil 75 saltaría al percentil 87.

\section{Análisis de sensibilidad}

Los resultados del análisis de sensibilidad se muestran en la tabla 3. Claramente, el análisis de sensibilidad que incluye los resultados en SIMCE 2004 y 2006 en el enfoque pre matching entrega un resultado extremo y que no hace mucho sentido $(0,40 \mathrm{DE})$. No parece viable que exista una variable omitida tan potente como el resultado de las pruebas anteriores. Sin embargo, aun incluyendo resultados de estas pruebas en el enfoque post matching, la diferencia encontrada seguiría siendo significativa. Como se explicó anteriormente, en este caso resulta más apropiado no incluir resultados de pruebas anteriores. Así, los resultados del análisis de sensibilidad son de 0,03 DE en la estimación post matching y 0,08 DE en la estimación pre matching. 
Tabla n. ${ }^{\circ}$ 3. Análisis de sensibilidad. Estimación del sesgo usando estimación pre y post matching

\begin{tabular}{|c|c|c|c|c|}
\hline Variable & $\begin{array}{c}\text { Pre matching } \\
\text { Coef. }\end{array}$ & Dif & $\begin{array}{c}\text { Post marching } \\
\text { Coef. }\end{array}$ & Dif \\
\hline SIMCE06 & 0,450 & 0,044 & 0,476 & 0,014 \\
\hline SIMCE06^2 & 0,057 & 0,447 & 0,055 & 0,004 \\
\hline SIMCE04 & 0,297 & 0,453 & 0,288 & 0,004 \\
\hline Ranking en el curso & 0,017 & 0,171 & 0,018 & 0,033 \\
\hline Repitente & 0,039 & 0,873 & 0,036 & 0,271 \\
\hline Libros en el hogar & 0,014 & 0,108 & 0,016 & 0,022 \\
\hline Mujer & 0,056 & 0,775 & 0,054 & 0,010 \\
\hline Escolaridad padres & 0,049 & 0,808 & 0,039 & 0,004 \\
\hline Escolaridad padres^2 & 0,076 & 0,188 & 0,058 & 0,005 \\
\hline Ypc & 0,026 & 0,203 & 0,011 & 0,004 \\
\hline $\mathrm{Ypc}^{\wedge} 2$ & 0,016 & 0,101 & 0,003 & 0,001 \\
\hline $\mathrm{Ypc}^{\wedge} 3$ & 0,008 & 0,036 & 0,009 & 0,249 \\
\hline Expect. TP & 0,010 & 0,220 & 0,006 & 0,028 \\
\hline Expect. TP superior & 0,003 & 0,631 & 0,009 & 0,006 \\
\hline Sin expectativas declaradas & 0,021 & 0,041 & 0,020 & 0,027 \\
\hline Expect. ed. media incompleta & 0,005 & 0,594 & 0,005 & 0,373 \\
\hline Expect. universitario & 0,053 & 0,336 & 0,060 & 0,076 \\
\hline Expect. posgrado & 0,051 & 0,347 & 0,051 & 0,047 \\
\hline Particular subvencionado & 0,027 & 0,240 & 0,026 & 0,008 \\
\hline Rural & 0,008 & 0,118 & 0,010 & 0,026 \\
\hline Promedio SIMCE 06 est. educ. & 0,084 & 0,440 & 0,081 & 0,351 \\
\hline N. ${ }^{\circ}$ de estudiantes est. educ. & 0,028 & 0,898 & 0,019 & 0,007 \\
\hline Promedio ISE por comuna & 0,023 & 0,057 & 0,023 & 0,216 \\
\hline Incluyendo test previos & & & & \\
\hline Valor máximo & 0,450 & 0,898 & 0,476 & 0,373 \\
\hline Sesgo estimado & \multicolumn{2}{|c|}{0,404} & \multicolumn{2}{|c|}{0,178} \\
\hline \multicolumn{5}{|l|}{ Excluyendo test previos } \\
\hline Valor máximo & 0,084 & 0,898 & 0,081 & 0,373 \\
\hline Sesgo estimado & \multicolumn{2}{|c|}{0,076} & \multicolumn{2}{|c|}{0,030} \\
\hline
\end{tabular}

Tomando una posición conservadora, se selecciona el escenario que afecta en mayor medida los resultados estimados para la brecha. En este caso el resultado reduciría las estimaciones en 0,08 DE. Esto implica que las estimaciones de brecha se moverían entre un 0,00 DE para los estudiantes con menor SIMCE 2006 a -0,41 DE para los estudiantes con mayor puntaje en la prueba SIMCE 2006. El promedio sería un efecto de -0,20 DE.

Este resultado es interesante al menos por dos razones. En primer lugar, incluso con un enfoque conservador, se sigue obteniendo una gran diferencia para los estudiantes con mayor capacidad. En segundo lugar, nos muestra que el resultado es relativamente estable 
para los distintos cuantiles, lo que es importante por el problema de autoselección en la PSU. Este análisis se complementa con el análisis de cotas y selección de muestra que se desarrolla más adelante.

\section{Especialidades}

En este caso se analizan las brechas para distintas especialidades en el ETP (ver tabla 4) comparadas con EHC. La menor brecha se obtiene para ETP Comercial y ETP Marítimo (-0,27 DE). Esto es esperable para comercial, debido a que esta especialidad incluye materias más cercanas a EHC, por ejemplo en contabilidad, ventas, administración y puestos de secretariado. No obstante, la diferencia sigue siendo elevada. La mayor diferencia aparece en la especialidad agrícola $(-0,35$ $\mathrm{DE})$, lo que también es esperable por un mayor trabajo en terreno.

Tabla n. ${ }^{\circ}$ 4. Efectos por especialidades. Post matching

\begin{tabular}{|l|c|c|}
\hline & M1 & M2 \\
\hline Comercial & $-0,254^{* *}$ & $-0,273^{* *}$ \\
& $(0,010)$ & $(0,010)$ \\
\hline Industrial & $-0,284^{* *}$ & $-0,304^{* *}$ \\
& $(0,012)$ & $(0,012)$ \\
\hline Mecánico & $-0,286^{* *}$ & $-0,319^{* *}$ \\
& $(0,011)$ & $(0,011)$ \\
\hline Agrícola & $-0,314^{* *}$ & $-0,346^{* *}$ \\
& $(0,020)$ & $(0,020)$ \\
\hline Marítimo & $-0,252^{* *}$ & $-0,269^{* *}$ \\
& $(0,035)$ & $(0,034)$ \\
\hline T x SIMCE06 & & $-0,072^{* *}$ \\
& & $(0,006)$ \\
\hline N & 102,150 & 102,150 \\
R-2 & 0,680 & 0,680 \\
\hline
\end{tabular}

Nota: Diferencias significativas entre grupos: $+\mathrm{p}<0,10,{ }^{*} \mathrm{p}<0,05,{ }^{* *} \mathrm{p}<0,01$. Errores estándar entre paréntesis. Errores agrupados a nivel del establecimiento. Los controles incluyen información personal, información académica, expectativas de los padres, información de las escuelas y de la comuna.

\section{Las diferencias en lenguaje y matemática}

Se encontró una mayor brecha en matemática (tabla 5). En este caso, el rango de variación del efecto en la PSU según puntaje en el SIMCE 2006 es de -0,09 DE hasta -0,56 DE. Las diferencias en lenguaje son más pequeñas, alcanzando un rango de variación del efecto entre -0,04 DE hasta -0,31 DE según su puntaje en el SIMCE 2006. 
Tabla n. ${ }^{\circ}$ 5. Regresiones de quantiles

\begin{tabular}{|l|c|c|c|c|c|}
\hline Quantiles & 20 & 40 & 60 & 80 & 100 \\
\hline T & $-0,253^{* *}$ & $-0,265^{* *}$ & $-0,275^{* *}$ & $-0,300^{* *}$ & $-0,326^{* *}$ \\
& $(0,006)$ & $(0,005)$ & $(0,004)$ & $(0,005)$ & $(0,047)$ \\
T*SIMCE06 & $-0,065^{* *}$ & $-0,071^{* *}$ & $-0,081^{* *}$ & $-0,087^{* *}$ & $-0,065+$ \\
& $(0,006)$ & $(0,005)$ & $(0,004)$ & $(0,005)$ & $(0,039)$ \\
\hline $\mathrm{N}$ & 103,325 & 103,325 & 103,325 & 103,325 & 103,325 \\
\hline
\end{tabular}

Nota: Diferencias significativas entre grupos: $+\mathrm{p}<0,10,{ }^{*} \mathrm{p}<0,05,{ }^{* *} \mathrm{p}<0,01$. Errores estándar entre paréntesis. Errores agrupados a nivel del establecimiento. Los controles incluyen información personal, información académica, expectativas de los padres, información de las escuelas y de la comuna.

En la tabla 6 se presentan los resultados de matemática y lenguaje para las diferentes especialidades ${ }^{8}$. Mientras comercial, industrial y marítima tienen el menor efecto en matemática, solo comercial y marítima presentan un bajo efecto para lenguaje. De hecho, industrial es una de las especialidades con una brecha mayor para lenguaje. Agrícola, por su parte, presenta las mayores brechas en ambas materias. Como es esperable, las distintas especialidades afectan la brecha en formas diversas.

Tabla n. ${ }^{\circ}$ 6. Diferencias para lenguaje y matemática, por especialidad

\begin{tabular}{|l|c|c|}
\hline Variable depend.: & PSU 2008 - mat. & PSU 2008 - leng. \\
& Ml & $-0,183^{* *}$ \\
\hline Comercial & $-0,291^{* *}$ & $(0,010)$ \\
& $(0,012)$ & $-0,242^{* *}$ \\
& $-0,290^{* *}$ & $(0,012)$ \\
\hline Industrial & $(0,014)$ & $-0,210^{* *}$ \\
& $-0,324^{* *}$ & $(0,011)$ \\
\hline Mecánico & $(0,014)$ & $-0,243^{* *}$ \\
& $-0,341^{* *}$ & $(0,019)$ \\
\hline Agrícola & $(0,027)$ & $-0,181^{* *}$ \\
& $-0,295^{* *}$ & $0,040)$ \\
\hline Marítimo & $(0,040)$ & 102,150 \\
& 102,150 & 0,620 \\
\hline $\mathrm{N}$ & 0,540 & \\
R-2 & \multicolumn{2}{|}{} \\
\hline
\end{tabular}

Nota: Diferencias significativas entre grupos: $+\mathrm{p}<0,10,{ }^{*} \mathrm{p}<0,05,{ }^{* *} \mathrm{p}<0,01$. Errores estándar entre paréntesis. Errores agrupados del establecimiento. Los controles incluyen información personal, información académica, expectativas de los padres, información de las escuelas y de la comuna. No considera variable de interacción.

8 Por simplicidad, en este caso las regresiones fueron realizadas sin incluir los efectos de interacción. 


\section{Selección de muestra}

Después de estudiar la diferencia de resultados en la PSU entre estudiantes de EHC y ETP, se analiza en mayor detalle el problema de autoselección en la PSU. En la tabla 1 se puede observar que en promedio el $43 \%$ de los estudiantes no toman la PSU inmediatamente después de la graduación. El porcentaje de estudiantes que no toman la prueba es mayor en ETP (60\%) que en EHC (24\%). Es evidente que la autoselección se produce a lo largo de toda la distribución del SIMCE 2006. Esto hace creer que si se encuentran contrapartes para cada uno de los estudiantes tratados, se tendrá una gran parte de la distribución cubierta. Sin embargo, como el porcentaje de estudiantes que no rinden la prueba es mayor en los deciles más bajos, el nivel de certeza disminuye en dichos deciles. La mayor certeza se da en los alumnos con mejor rendimiento, que es el rango más interesante para analizar debido al potencial efecto en el acceso a educación superior.

La figura 6 (ver anexo) muestra una cierta homogeneidad en los quintiles cuando se compara los estudiantes que toman la prueba y los que no.

Por último, la tabla 7 presenta el ejercicio de poner cotas a los resultados obtenidos anteriormente. En la mayoría de los casos se encuentran cotas razonables para las estimaciones. Los percentiles $10 \mathrm{y}$ 70 presentan algunas incongruencias en los resultados, pero se puede atribuir a la pequeña muestra en cada decil y a la cercanía con el valor estimado. Como se presenta, si en cada decil se eliminan los alumnos con menor rendimiento en el SIMCE 2006 de EHC hasta tener la misma proporción de estudiantes que ETP, las diferencias tienden a aumentar. Por otra parte, si se eliminan los alumnos con mejores resultados, se obtiene una diferencia menor. Ambas cotas están muy cerca de la brecha encontrada en cada decil, lo que confirma la estabilidad de los resultados ${ }^{9}$. Al igual que en las regresiones de cuantiles, cuando se modifica la muestra para obtener cotas superiores e inferiores no se aprecia gran variación en los resultados. Esto indica que, si toda

9 En resultados no reportados, realizamos un modelo de selección de Heckman obteniendo resultados similares. 
la población rindiera la PSU, probablemente se obtendrían resultados similares a los obtenidos en este estudio.

Tabla n. ${ }^{\circ}$ 7. Cotas superior e inferior para el efecto del tratamiento en 5 deciles del SIMCE 2006. Muestra 2

\begin{tabular}{|l|c|c|c|c|c|c|c|c|c|}
\hline Decil & & 10 & & & 30 & & & 50 & \\
\hline Cota & Inferior & Real & Superior & Inferior & Real & Superior & Inferior & Real & Superior \\
\hline T & $-0,153^{* *}$ & $-0,144^{* *}$ & $-0,152^{* *}$ & $-0,175^{* *}$ & $-0,185^{* *}$ & $-0,202^{* *}$ & $-0,218^{* *}$ & $-0,229^{* *}$ & $-0,234^{* *}$ \\
& $(0,017)$ & $(0,016)$ & $(0,017)$ & $(0,014)$ & $(0,013)$ & $(0,014)$ & $(0,012)$ & $(0,010)$ & $(0,011)$ \\
\hline N & 4,358 & 5,030 & 4,464 & 6,051 & 7,140 & 6,118 & 8,146 & 9,869 & 8,180 \\
R-2 & 0,190 & 0,210 & 0,220 & 0,210 & 0,230 & 0,230 & 0,230 & 0,250 & 0,250 \\
\hline Decil & & 70 & & & 90 & & & & \\
\hline Cota & Inferior & Real & Superior & Inferior & Real & Superior & & & \\
\hline T & $-0,272^{* *}$ & $-0,266^{* *}$ & $-0,265^{* *}$ & $-0,318^{* *}$ & $-0,326^{* *}$ & $-0,336^{* *}$ & & & \\
& $(0,009)$ & $(0,008)$ & $(0,010)$ & $(0,008)$ & $(0,008)$ & $(0,008)$ & & & \\
\hline N & 10,208 & 12,641 & 10,302 & 12,737 & 15,037 & 12,594 & & & \\
R-2 & 0,280 & 0,290 & 0,310 & 0,380 & 0,420 & 0,440 & & & \\
\hline
\end{tabular}

Nota: Diferencias significativas entre grupos: $+\mathrm{p}<0,10,{ }^{*} \mathrm{p}<0,05,{ }^{* *} \mathrm{p}<0,01$. Errores estándar entre paréntesis.

Errores agrupados del establecimiento. Los controles incluyen información personal, información académica, expectativas de los padres, información de las escuelas y de la comuna. El efecto de la interacción entre el tratamiento y el puntaje SIMCE 2006 no fue incluido en este análisis. Para estimar la banda inferior, se eliminan estudiantes de EHC con alto rendimiento en SIMCE 2006 hasta equiparar la proporción de estudiantes ETP presentes en cada decil de puntajes SIMCE 2006. Para estimar la cota superior se repite el procedimiento pero esta vez se eliminan a los alumnos con peores rendimientos.

\section{Discusión y limitaciones del estudio}

En este estudio se comparó el resultado académico de la ETP y la EHC en Chile y se encontró que estudiantes similares que eligen ETP obtienen en promedio 0,28 DE menos en la PSU que los estudiantes que eligen EHC. Esta diferencia es mayor para estudiantes con altos puntajes en el SIMCE de segundo medio y es pequeña para estudiantes con bajos resultados en el mismo SIMCE. La diferencia para estudiantes de alto rendimiento en ETP alcanza las 0,56 DE en matemática y 0,31 DE en lenguaje. Los resultados son similares a los encontrados en la literatura sobre tracks en las escuelas en los EE.UU. (Gamoran, 1987; Gamoran, 1989; Gamoran y Mare, 1989, y Carbonaro, 2005). La brecha también varía por especialidad.

Los resultados muestran que los estudiantes con mejor rendimiento, con aspiraciones de ingresar a la universidad y que eligen 
ir a una ETP pueden estar reduciendo sus posibilidades de acceso a la educación superior o de ir a una carrera o universidad de mayor prestigio. Esta reducción de posibilidades también podría darse en el acceso a becas o créditos con menor tasa de interés.

Por otra parte, el impacto académico en aquellos estudiantes con menor rendimiento que se matriculan en ETP parece ser pequeño. La ETP podría tener efectos positivos si aumenta efectivamente la empleabilidad. No sería cierto para este grupo, que la provisión de educación técnica estaría reduciendo su capital humano, comparado con similares estudiantes que asisten a EHC. No obstante, los altos niveles de autoselección en la PSU y posibles debilidades del instrumento para capturar diferencias confiables en la parte baja de la distribución de resultados, sugieren ser cautos en las conclusiones que se pueden extraer para este grupo.

Los resultados de los quintiles superiores son más sólidos. El efecto de la motivación de los estudiantes al momento de rendir la PSU podrían afectar en alguna forma no observable los resultados. Sin embargo, se cree que al controlar por nivel socioeconómico, resultados de las pruebas anteriores y expectativas, se reduce bastante su efecto. Es altamente probable que el análisis de sensibilidad dé cuenta adecuada del efecto restante. Más aún, los resultados podrían verse afectados por un sesgo a la baja debido a que son los estudiantes de mayor rendimiento en la ETP los que rinden la PSU.

Estos resultados son interesantes principalmente por dos razones. Primero, desde un punto de vista de eficiencia económica, ellos ayudan a entender de manera más comprehensiva los efectos de la ETP en un país como Chile, donde una alta proporción de estudiantes secundarios eligen ETP. Estas estimaciones permiten un juicio mejor fundado sobre los costos de elegir ETP, en particular para los estudiantes con mayor rendimiento. Aunque no es posible extraer conclusiones generales sobre el valor de la ETP comparado al valor de la EHC, en particular sin considerar una posible ventaja relativa de la ETP en la retención de estudiantes o en una mayor empleabilidad, los resultados sugieren que el valor de la ETP podría ser muy bajo e incluso negativo para los estudiantes con mayor rendimiento académico. 
Los estudiantes con mayores habilidades académicas que eligen ETP podrían estar adquiriendo considerablemente menos capital humano que el que hubiesen adquirido si hubieran elegido EHC. Estos mismos estudiantes podrían también estar limitando sus oportunidades educacionales -en términos de acceso y de financiamiento-así como sus ingresos futuros. Esto podría tener consecuencias negativas para la economía chilena, debido a que podría reducir la productividad de una porción importante de jóvenes.

Segundo, desde el punto de vista de la equidad, este estudio levanta el tema de la educación como un instrumento de estratificación en la sociedad chilena. El gobierno promovió la expansión de la ETP en las décadas de los 80 y 90 con el objetivo de proveer una educación media que preparara para ingresar al mercado laboral a los estudiantes de menor nivel socioeconómico, los que normalmente tenían bajo rendimiento académico en educación básica (en parte debido a una educación de menor calidad). No obstante, muchos estudiantes con altas habilidades académicas también ingresaron a la ETP. En países altamente estratificados como Chile, la EHC es aún vista por las familias de nivel socioeconómico bajo o medio-bajo como educación para jóvenes de clase social más alta, no para sus hijos. Los resultados sugieren que estas visiones combinadas con un menor valor agregado por la ETP para los estudiantes con mayores habilidades académicas de bajo nivel socioeconómico, parecen estar perpetuando las inequidades sociales en Chile.

¿Por qué estudiantes con altas expectativas y buen rendimiento eligen ETP? Debido a que las aspiraciones por educación universitaria han crecido fuertemente en los últimos años entre los alumnos de menor nivel socioeconómico, ¿ipor qué las elecciones de los estudiantes y sus familias no se han ajustado a estos cambios en sus aspiraciones? En Chile las decisiones tempranas parecen tener profundos efectos en las oportunidades futuras. Ciertamente, se requiere más investigación para entender completamente este fenómeno.

Damos las gracias al Ministerio de Educación de Chile, en particular al Centro de Estudios y al SIMCE, por el apoyo y la información proporcionada; a Ana María Baeza, Gastón Bravo y 
Francisco Lagos, como también al Comité Técnico Asesor de la PSU por la información proporcionada. A Martin Carnoy, Susana Loeb, Sean Reardon, Eric Bettinger, Anthony Antonio y María Pérez, participantes del seminario IREPP de la Universidad de Stanford, y a Patrick McEwan, Cristián Aedo, María José Ramírez y Sergio Celis, por sus comentarios y apoyo.

Las ideas expresadas aquí son de exclusiva responsabilidad de sus autores.

\section{Referencias bibliográficas}

Ananda, P.; Mizala A.; Repetto, A. (2009). Using school scholarships to estimate the effect of private education on the academic achievement of low-income students in Chile. Economics of Education Review 28, 370-381.

Angrist, J.; Bettinger, E.; Kremer, M. (2004). Long-term consequences of secondary school vouchers: Evidence from administrative records in Colombia. Working Paper 10713. Obtenido en http://www.nber.org/ papers/w10713

Arum, R.; Shavit, Y. (1995). Secondary vocational education and the transition from school to work. Sociology of Education, vol. 68, n. ${ }^{\circ}$ 3, 187-204. American Sociological Association.

Blossfeld, H.P.; Shavit, Y. (2000). Persisting barriers: Changes in educational opportunities. In Arum, R. y Beattie, I. (eds.). The structure of schooling. Mountain View: Mayfield.

Caliendo, M.; Kopeinig, S. (2008). Some practical guidance for the implementation of propensity score matching. Journal of Economic Surveys, 22 (1), 31-72.

Carnoy, M. (2007). Cuba's academic advantage. Stanford University Press. Stanford, California.

Carnoy, M. (2008). Vocational education and training in the new global economy. Mimeo. Stanford University.

Centro de Microdatos (2009). Estudio de seguimiento de egresados de la EMTP. Obtenido en http://www.formaciontecnica.cl/archivosNoticias/(10) Presentacion-Seguimiento-de-egresados-EMTP.ppt.

Coleman, J. (1988). Social capital in the creation of human capital. American Journal of Sociology 94, Supplement: S95-S120. 
Comisión para el estudio de la formación técnico-profesional en Chile. (2009). Bases para una politica de formación técnico-profesional en Chile. Informe ejecutivo.

Comité Técnico Asesor. (2010). Resultados de la vinculación Prueba SIMCE 2006 y PSU Admisión 2009. http://www.consejoderectores.cl/site/pdf/ spsu.pdf

Dehejia, R.; Wahba, S. (2002). Propensity score matching methods for nonexperimental causal studies. The Review of Economics and Statistics, 84/1,151-161.

Drori, G.; Meyer, J.W.; Ramirez, F.O.; Schofer, E. (2003). Science in the modern world polity. Stanford University Press. Part Two: The Global Field of Science, 77-116. Part IV. The Impact of Science Globalization, 217-310.

Duflo, E.; Glennerster, R.; Kremer, M. (2007). Using randomization in development economics research: A toolkit. CEPR Working Paper 6059.

Gamoran, A. (1987). The stratification of high school learning opportunities. Sociology of Education 60, 135-55.

Hallinan, M.; Kubitschek, W. N. (1999). Curriculum differentiation and high school achievement. Social Psychology of Education, 3, 41-62.

Hallinan, M. T. (1994). Tracking: From theory to practice. Sociology of Education, vol. 67, n. ${ }^{\circ}$ 2, 79-84.

Hanushek, E. A.; Wößmann, L. (2006). Does educational tracking affect performance and inequality? Differences-in-differences evidence across countries. Economic Journal, 116 (510), 63-76.

Heckman, J. (1979). Sample selection bias as a specification error. Econometrica 47, 153-161.

Kelly, S.; Price, H. (2009). Vocational education: A clean slate for disengaged students? Social Science Research 38, 810-825.

Lucas, S. R. (1999). Tracking inequality: Stratification and mobility in American high schools. New York: Teachers College Press.

Meckes, L. y Carrasco, R. (2010). Two Decades of SIMCE: An overview of the National Assessment System in Chile. Assessment in Education: Principles, Policy y Practice 17, n. ${ }^{\circ}$ 2, 16.

Ministerio de Educación (2010). Indicadores de la Educación en Chile $2007-$ 2008.

Oakes, J. (1985). Keeping track: How schools structure inequality. New Haven, CT: Yale University Press. 
OCED (2008a). Costs and benefits in vocational education and training. Paris.

OCED (2008b). Education at a glance 2008. Paris.

Reardon, S.F.; Cheadle, J.E.; Robinson, J.P. (2009). The effect of catholic schooling on math and reading development in kindergarten through fifth grade. Journal of Research on Educational Effectiveness, 2, 45-87.

Reardon, S.F. (2010). Applied quasiexperimental research in education. Lecture notes. Stanford.

Rosenbaum, P.R. (1986). Dropping out of high school in the United States: An observational study. Journal of Educational Statistics, 11/3:207-224.

Schofer, E.; Meyer, J.W. (2005). The world-wide expansion of higher education in the Twentieth Century. American Sociological Review 70, 898-920.

Symonds, W.; Schwartz, R.B.; Ferguson, R. (2011). Pathways to prosperity: Meeting the challenge of preparing young Americans for the 21st Century. Report issued by the Pathways to Prosperity Project, Harvard Graduate School of Education.

Vanfossen, B.; Jones, J.; Spade, J. (1987). Curriculum tracking and status maintenance. Sociology of Education 60, 104-22.

Recibido: 4/10/2011

Aceptado: 21/02/2012 


\section{Anexo}

Figura 2: Región de soporte común

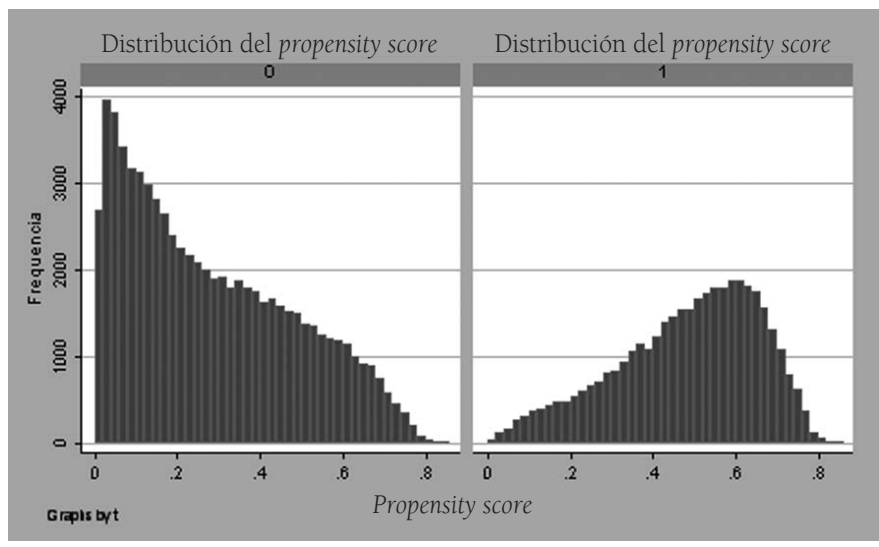

Nota: 0: estudiantes in EHC; 1: estudiantes en ETP.

Figura 3: Evaluación del matching: Propensity score pre y post matching

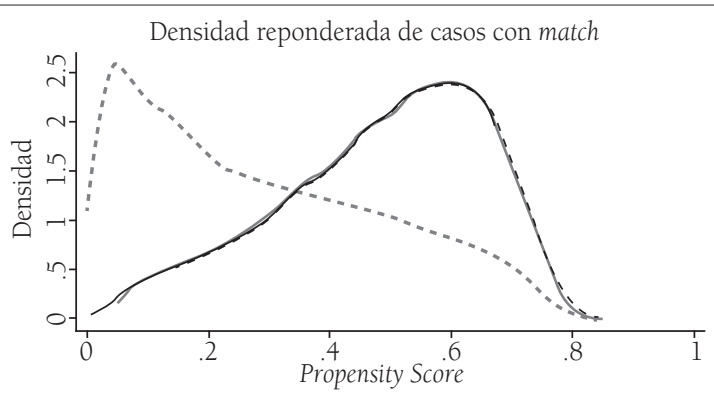

$$
\begin{aligned}
& \text { Casos del tratamiento con match } \\
& \text { - Casos del control usados como match (reponderados) } \\
& \text { - - - - - - Muestra total de casos tratados }
\end{aligned}
$$


118 DIFERENCIAS EN RESULTADOS ACADÉMICOS ENTRE EDUCACIÓN TÉCNICOPROFESIONAL Y HUMANISTA-CIENTÍFICA EN CHILE - M. Farías, R. Carrasco

Figura 4: Evaluando el balance: Densidad de la escolaridad de los padres pre y post matching

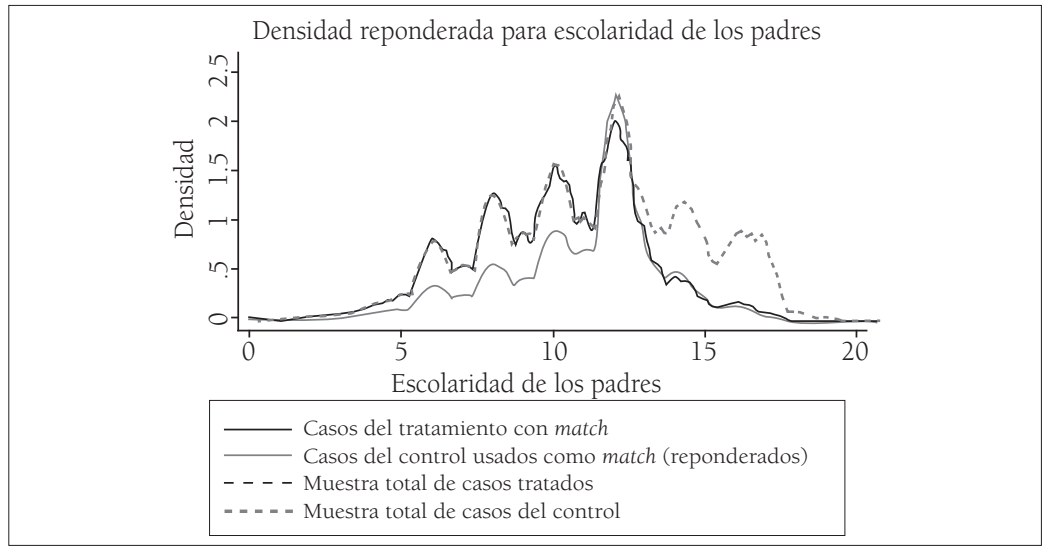

Figura 5: Densidad reponderada de los grupos de tratamiento y control para la PSU 2008

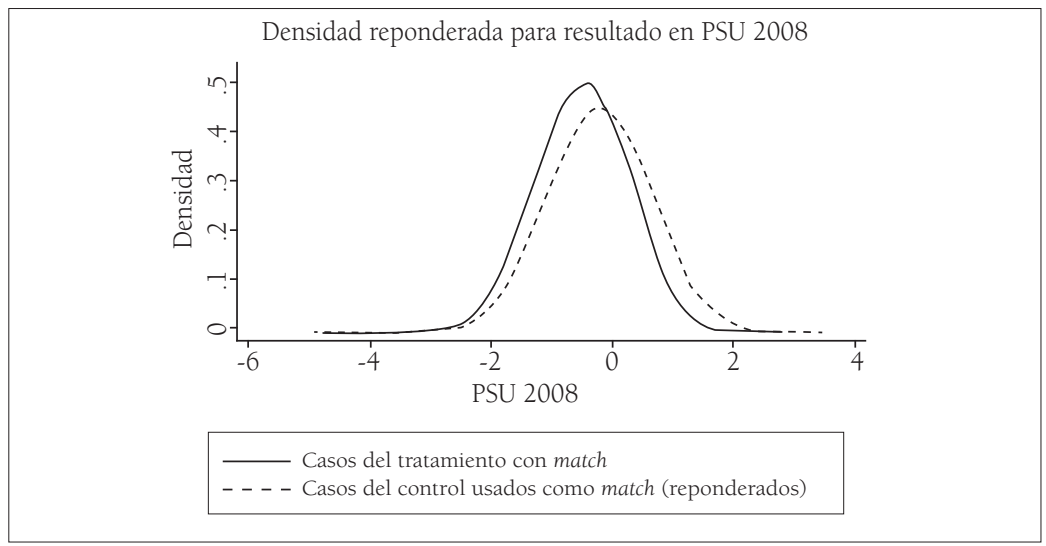


Tabla n. ${ }^{\circ}$ 8. Estadísticas

\begin{tabular}{|l|c|c|c|c|c|}
\hline Nombre de variable & Obs. & Media & Desv. est. & Min. & Max. \\
\hline TP & 113,274 & 0,354 & 0,478 & 0,000 & 1,000 \\
\hline Información académica & \multicolumn{5}{|l|}{} \\
\hline Promedio de la PSU 2009 (estandarizada) & 113,274 & 0,000 & 1,000 & $-4,923$ & 3,543 \\
\hline Promedio del. SIMCE 2006(estand.) & 113,274 & 0,000 & 1,000 & $-3,283$ & 2,882 \\
\hline Promedio del SIMCE 2004 (estand.) & 113,274 & 0,000 & 1,000 & $-3,726$ & 3,042 \\
\hline Ranking en el curso en 2006 & 113,274 & 16,64 & 10,78 & 1,000 & 48,00 \\
\hline Información personal & 113,252 & 0,551 & 0,497 & 0,000 & 1,000 \\
\hline Mujer (0: Hombre 1: Mujer) & 112,102 & 11,44 & 3,235 & 0,000 & 21,00 \\
\hline Escolaridad promedio de los padres 2006 & 111,230 & 0,000 & 1,000 & $-0,981$ & 17,21 \\
\hline Ingreso per cápita 2006 (estand.) & 112,088 & 29,18 & 34,21 & 0,000 & 201,0 \\
\hline Número de libros en el hogar 2006 & 112,057 & 0,064 & 0,246 & 0,000 & 1,000 \\
\hline Repitente & 113,036 & 0,596 & 0,491 & 0,000 & 1,000 \\
\hline Información establecimiento escolar & 113,274 & 0,090 & 0,286 & 0,000 & 1,000 \\
\hline Privado subvencionado & 113,274 & 196,9 & 144,3 & 4,000 & 774,0 \\
\hline Rural & 113,274 & 0,000 & 0,604 & $-1,976$ & 1,617 \\
\hline Número estudiantes del establecimiento & 113,274 & 0,002 & 0,045 & 0,000 & 1,000 \\
\hline Promedio SIMCE 2006 (estand.) & 113,274 & 0,045 & 0,208 & 0,000 & 1,000 \\
\hline Expectativas de los padres & 113,274 & 0,104 & 0,305 & 0,000 & 1,000 \\
\hline Educación media incomplete & 113,274 & 0,139 & 0,346 & 0,000 & 1,000 \\
\hline Título educación media TP & 113,274 & 0,442 & 0,497 & 0,000 & 1,000 \\
\hline Título educación media HC & 113,274 & 0,088 & 0,283 & 0,000 & 1,000 \\
\hline Título educación técnica superior & 113,274 & 0,180 & 0,384 & 0,000 & 1,000 \\
\hline Título universitario & 112,381 & 0,171 & 0,698 & $-2,569$ & 2,290 \\
\hline Posgrado & Expectativas no declaradas &
\end{tabular}

Tabla n. ${ }^{\circ}$ 9. Estimación de efectos de ETP. Regresiones con controles, post matching

\begin{tabular}{|l|c|c|c|c|}
\hline & \multicolumn{2}{|c|}{ MCO } & \multicolumn{2}{c|}{ Matching } \\
\hline Variable dependiente: PSU 08 & M1 & M2 & M3 & M4 \\
\hline T & $\begin{array}{r}0,280^{* *} \\
(0,009)\end{array}$ & $\begin{array}{c}0,291^{* *} \\
(0,008)\end{array}$ & $\begin{array}{c}0,257^{* *} \\
(0,009)\end{array}$ & $\begin{array}{c}0,282^{* *} \\
(0,009)\end{array}$ \\
\hline T x SIMCE06 & & $\begin{array}{c}0,054^{* *} \\
(0,005)\end{array}$ & & $\begin{array}{c}0,075^{* *} \\
(0,006)\end{array}$ \\
\hline $\mathrm{N}$ & 107,968 & 107,966 & 103,272 & 103,272 \\
\hline R-2 & 0,770 & 0,770 & 0,670 & 0,680 \\
\hline Controles & \multicolumn{5}{|l}{} \\
\hline Información personal & $\mathrm{x}$ & $\mathrm{x}$ & $\mathrm{x}$ & $\mathrm{x}$ \\
\hline Información académica & $\mathrm{x}$ & $\mathrm{x}$ & $\mathrm{x}$ & $\mathrm{x}$ \\
\hline Expectativas de los padres & $\mathrm{x}$ & $\mathrm{x}$ & $\mathrm{x}$ & $\mathrm{x}$ \\
\hline Información del establecimiento & $\mathrm{x}$ & $\mathrm{x}$ & $\mathrm{x}$ & $\mathrm{x}$ \\
\hline Información de la comuna & $\mathrm{x}$ & $\mathrm{x}$ & $\mathrm{x}$ & $\mathrm{x}$ \\
\hline
\end{tabular}

Nota: Diferencias significativas entre grupos: $+\mathrm{p}<0,10,{ }^{*} \mathrm{p}<0,05,{ }^{* *} \mathrm{p}<0,01$. Errores estándar entre paréntesis. Errores agrupados a nivel del establecimiento. 
Figura 6: Comparación de las densidades reponderadas en cuatro deciles del SIMCE 2006 entre los grupos de tratamiento y control, según si rindieron o no la PSU.
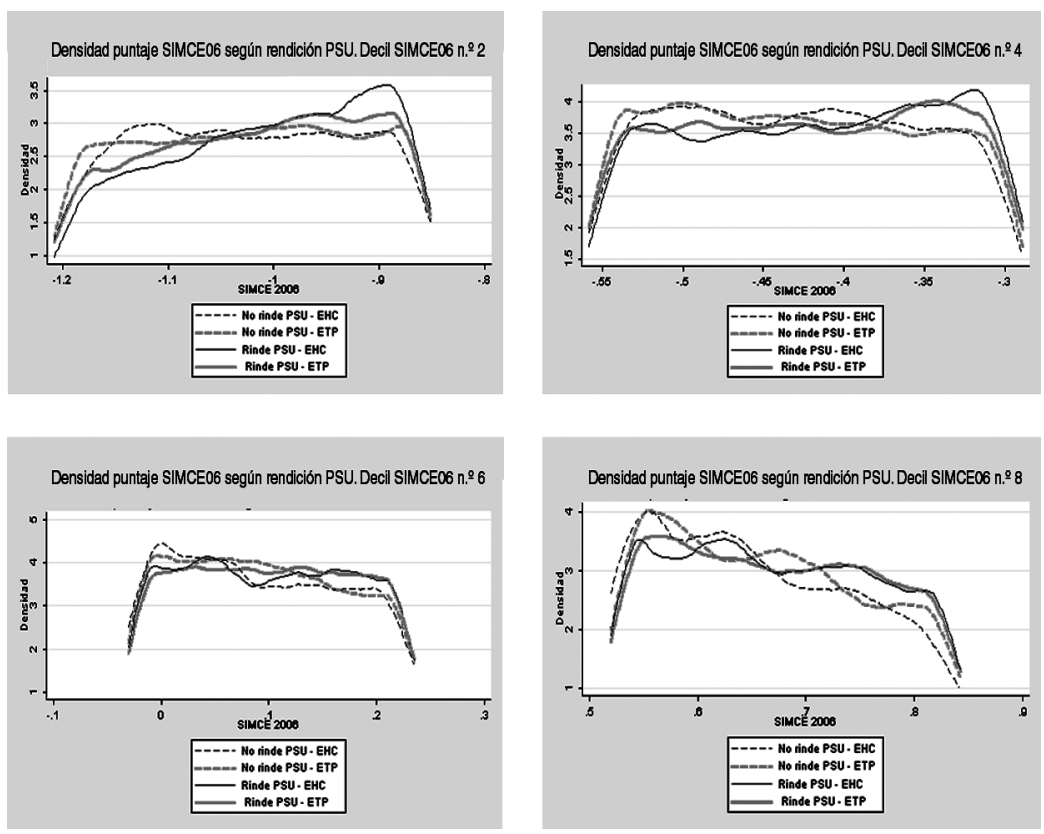

Tabla n. ${ }^{\circ}$ 10. Diferencias para lenguaje y matemática

\begin{tabular}{|l|c|c|c|c|}
\hline \multirow{2}{*}{ Variable dependiente: } & \multicolumn{2}{|c|}{ PSU 2008 - matemática } & \multicolumn{2}{c|}{ PSU 2008 - lenguaje } \\
\cline { 2 - 5 } & M1 & M2 & M3 & M4 \\
\hline T & $-0,284^{* *}$ & $-0,311^{* *}$ & $-0,197^{* *}$ & $-0,208^{* *}$ \\
& $(0,010)$ & $(0,010)$ & $(0,009)$ & $(0,009)$ \\
\hline T x SIMCE06 Mat & & $-0,090^{* *}$ & & \\
& & $(0,007)$ & & $-0,035^{* *}$ \\
\hline T x SIMCE06 Leng & & & & $(0,005)$ \\
\hline N & & & & 103,272 \\
R-2 & 103,272 & 103,272 & 103,272 & 0,620 \\
\hline
\end{tabular}

Nota: Diferencias significativas entre grupos: $+\mathrm{p}<0,10,{ }^{*} \mathrm{p}<0,05,{ }^{* *} \mathrm{p}<0,01$. Errores estándar entre paréntesis. Errores agrupados a nivel del establecimiento. Los controles incluyen información personal, información académica, expectativas de los padres, información de las escuelas y de la comuna. 
Tabla n. ${ }^{\circ}$ 11. Estimación de efectos de ETP. Detalle tabla 5

\begin{tabular}{|c|c|c|c|c|}
\hline \multirow[b]{2}{*}{ Variable dependiente: PSU 08} & \multicolumn{2}{|c|}{$\mathrm{MCO}$} & \multicolumn{2}{|c|}{ Matching } \\
\hline & Ml & M2 & M3 & M4 \\
\hline $\mathrm{T}$ & $\begin{array}{c}-0,280^{* *} \\
(0,009) \\
\end{array}$ & $\begin{array}{c}-0,291^{* *} \\
(0,008)\end{array}$ & $\begin{array}{c}-0,257^{* *} \\
(0,009) \\
\end{array}$ & $\begin{array}{c}-0,282^{* *} \\
(0,009) \\
\end{array}$ \\
\hline T x SIMCE06 & & $\begin{array}{c}-0,054^{* *} \\
(0,005)\end{array}$ & & $\begin{array}{c}-0,075^{* *} \\
(0,006) \\
\end{array}$ \\
\hline SIMCE06 & $\begin{array}{c}0,442^{* *} \\
(0,007)\end{array}$ & $\begin{array}{c}0,457^{* *} \\
(0,007)\end{array}$ & $\begin{array}{c}0,455^{* *} \\
(0,008)\end{array}$ & $\begin{array}{c}0,490 * * \\
(0,008)\end{array}$ \\
\hline $\mathrm{SIMCEO}^{\prime \wedge} 2$ & $\begin{array}{c}0,053^{* *} \\
(0,002)\end{array}$ & $\begin{array}{l}0,047^{* * *} \\
(0,002)\end{array}$ & $\begin{array}{l}0,048^{* *} \\
(0,002)\end{array}$ & $\begin{array}{c}0,048^{* *} \\
(0,002)\end{array}$ \\
\hline SIMCE04 & $\begin{array}{c}0,305^{* *} \\
(0,004) \\
\end{array}$ & $\begin{array}{c}0,306^{* *} \\
(0,004)\end{array}$ & $\begin{array}{c}0,296^{* *} \\
(0,005)\end{array}$ & $\begin{array}{c}0,295^{* *} \\
(0,005) \\
\end{array}$ \\
\hline Ranking en el curso & $\begin{array}{c}0,002^{* *} \\
(0,000)\end{array}$ & $\begin{array}{l}0,002^{* *} \\
(0,000)\end{array}$ & $\begin{array}{c}0,002^{* *} \\
(0,000) \\
\end{array}$ & $\begin{array}{c}0,002 * * \\
(0,000) \\
\end{array}$ \\
\hline Repitente & $\begin{array}{c}-0,127^{* *} \\
(0,007)\end{array}$ & $\begin{array}{c}-0,127^{* *} \\
(0,007)\end{array}$ & $\begin{array}{c}-0,121 * * \\
(0,009)\end{array}$ & $\begin{array}{c}-0,120 * * \\
(0,009)\end{array}$ \\
\hline Libros en el hogar & $\begin{array}{c}0,000^{* *} \\
(0,000)\end{array}$ & $\begin{array}{c}0,000 * * \\
(0,000)\end{array}$ & $\begin{array}{c}0,001^{* *} \\
(0,000)\end{array}$ & $\begin{array}{c}0,001 * * \\
(0,000)\end{array}$ \\
\hline Mujer & $\begin{array}{c}-0,109 * * \\
(0,005) \\
\end{array}$ & $\begin{array}{c}-0,110^{* *} \\
(0,005) \\
\end{array}$ & $\begin{array}{c}-0,101^{* *} \\
(0,006)\end{array}$ & $\begin{array}{c}-0,102^{* *} \\
(0,006) \\
\end{array}$ \\
\hline Escolaridad padres & $\begin{array}{c}-0,015^{* *} \\
(0,003)\end{array}$ & $\begin{array}{c}-0,014^{* *} \\
(0,003)\end{array}$ & $\begin{array}{c}-0,014^{* *} \\
(0,004)\end{array}$ & $\begin{array}{c}-0,015^{* *} \\
(0,004) \\
\end{array}$ \\
\hline Escolaridad padres ${ }^{\wedge} 2$ & $\begin{array}{c}0,001^{* * *} \\
(0,000)\end{array}$ & $\begin{array}{c}0,001^{* *} \\
(0,000)\end{array}$ & $\begin{array}{l}0,001^{* *} \\
(0,000)\end{array}$ & $\begin{array}{c}0,001^{* *} \\
(0,000) \\
\end{array}$ \\
\hline Ypc & $\begin{array}{c}0,043 * * \\
(0,004)\end{array}$ & $\begin{array}{c}0,042^{* *} \\
(0,004)\end{array}$ & $\begin{array}{c}0,031^{* *} \\
(0,006)\end{array}$ & $\begin{array}{c}0,031^{* *} \\
(0,006) \\
\end{array}$ \\
\hline $\mathrm{Ypc}^{\wedge} 2$ & $\begin{array}{c}-0,007^{* *} \\
(0,002)\end{array}$ & $\begin{array}{c}-0,007^{* *} \\
(0,002)\end{array}$ & $\begin{array}{l}-0,005 \\
(0,005)\end{array}$ & $\begin{array}{l}-0,006 \\
(0,005)\end{array}$ \\
\hline $\mathrm{Ypc}^{\wedge} 3$ & $\begin{array}{l}0,000+ \\
(0,000)\end{array}$ & $\begin{array}{c}0,000 \\
(0,000)\end{array}$ & $\begin{array}{c}0,000 \\
(0,001)\end{array}$ & $\begin{array}{c}0,000 \\
(0,001)\end{array}$ \\
\hline Expect. TP & $\begin{array}{c}-0,033^{* *} \\
(0,009) \\
\end{array}$ & $\begin{array}{l}-0,020^{*} \\
(0,009)\end{array}$ & $\begin{array}{c}0,017 \\
(0,014) \\
\end{array}$ & $\begin{array}{c}0,018 \\
(0,014) \\
\end{array}$ \\
\hline Expect. TP superior & $\begin{array}{c}-0,003 \\
(0,007) \\
\end{array}$ & $\begin{array}{c}0,005 \\
(0,006)\end{array}$ & $\begin{array}{c}0,012 \\
(0,007)\end{array}$ & $\begin{array}{c}0,019 * * \\
(0,007) \\
\end{array}$ \\
\hline Expect. ed. media incompleta & $\begin{array}{c}0,06 \\
(0,040)\end{array}$ & $\begin{array}{l}0,069+ \\
(0,040)\end{array}$ & $\begin{array}{c}0,055 \\
(0,043)\end{array}$ & $\begin{array}{c}0,071 \\
(0,043) \\
\end{array}$ \\
\hline Expect. universitario & $\begin{array}{c}0,107^{* *} \\
(0,006) \\
\end{array}$ & $\begin{array}{c}0,113^{* *} \\
(0,006)\end{array}$ & $\begin{array}{l}0,122^{* *} \\
(0,007) \\
\end{array}$ & $\begin{array}{c}0,125^{* *} \\
(0,007) \\
\end{array}$ \\
\hline Expect. posgrado & $\begin{array}{c}0,196^{* * *} \\
(0,008)\end{array}$ & $\begin{array}{c}0,199^{* *} \\
(0,008)\end{array}$ & $\begin{array}{c}0,200^{* *} \\
(0,011)\end{array}$ & $\begin{array}{c}0,199 * * \\
(0,010)\end{array}$ \\
\hline Sin expectativas declaradas & $\begin{array}{c}0,054^{* * *} \\
(0,007)\end{array}$ & $\begin{array}{c}0,059^{* *} \\
(0,007)\end{array}$ & $\begin{array}{c}0,048^{* *} \\
(0,008)\end{array}$ & $\begin{array}{c}0,053^{* *} \\
(0,008)\end{array}$ \\
\hline Particular subvencionado & $\begin{array}{c}0,054^{* * *} \\
(0,009)\end{array}$ & $\begin{array}{c}0,055^{* *} \\
(0,009)\end{array}$ & $\begin{array}{l}0,052^{* *} \\
(0,009) \\
\end{array}$ & $\begin{array}{c}0,053^{* *} \\
(0,009) \\
\end{array}$ \\
\hline Rural & $\begin{array}{c}-0,028^{* *} \\
(0,007) \\
\end{array}$ & $\begin{array}{c}-0,027^{* *} \\
(0,007) \\
\end{array}$ & $\begin{array}{c}-0,033 * * \\
(0,008)\end{array}$ & $\begin{array}{c}-0,031^{* * *} \\
(0,008) \\
\end{array}$ \\
\hline Promedio SIMCE06 est. educ. & $\begin{array}{c}0,146^{* *} \\
(0,010)\end{array}$ & $\begin{array}{c}0,146^{* *} \\
(0,010)\end{array}$ & $\begin{array}{l}0,138^{* *} \\
(0,011)\end{array}$ & $\begin{array}{c}0,139 * * \\
(0,011)\end{array}$ \\
\hline N. ${ }^{\circ}$ de estudiantes est. educ. & $\begin{array}{c}0,027 * * \\
(0,006) \\
\end{array}$ & $\begin{array}{c}0,028^{* * *} \\
(0,006)\end{array}$ & $\begin{array}{c}0,018^{* *} \\
(0,006) \\
\end{array}$ & $\begin{array}{r}0,019 * * \\
(0,006) \\
\end{array}$ \\
\hline Promedio NSE por comuna & $\begin{array}{c}0,034^{* *} \\
(0,005)\end{array}$ & $\begin{array}{l}0,033^{* *} \\
(0,005)\end{array}$ & $\begin{array}{l}0,034 * * \\
(0,005)\end{array}$ & $\begin{array}{c}0,033^{* *} \\
(0,005) \\
\end{array}$ \\
\hline Constant. & $\begin{array}{c}-0,041^{*} \\
(0,018)\end{array}$ & $\begin{array}{c}-0,042^{*} \\
(0,018)\end{array}$ & $\begin{array}{c}-0,074 * * \\
(0,021)\end{array}$ & $\begin{array}{c}-0,058^{* *} \\
(0,021)\end{array}$ \\
\hline $\begin{array}{l}\mathrm{N} \\
\mathrm{R}-2 \\
\end{array}$ & $\begin{array}{c}107,966 \\
0,770 \\
\end{array}$ & $\begin{array}{c}107,966 \\
0,770 \\
\end{array}$ & $\begin{array}{c}103,272 \\
0,670 \\
\end{array}$ & $\begin{array}{c}103,272 \\
0,680\end{array}$ \\
\hline
\end{tabular}

Nota: Diferencias significativas entre grupos: $+\mathrm{p}<0,10,{ }^{*} \mathrm{p}<0,05,{ }^{* *} \mathrm{p}<0,01$. Errores estándar entre paréntesis. Errores agrupados del establecimiento 